\title{
Approximation of the Electron Density of Aluminium Clusters in Tensor-Product Format
}

\author{
T. Blesgen ${ }^{\mathrm{a}, *}$, V. Gavini ${ }^{\mathrm{b}}$, V. Khoromskaia ${ }^{\mathrm{c}}$ \\ ${ }^{a}$ Max-Planck-Institute for Mathematics in the Sciences, \\ Inselstraße 22-26, D-04103 Leipzig \\ ${ }^{b}$ Department of Mechanical Engineering, University of Michigan, MI 48109-2125, USA \\ ${ }^{c}$ Max-Planck-Institute for Mathematics in the Sciences, \\ Inselstraße 22-26, D-04103 Leipzig
}

\begin{abstract}
The tensor-structured methods developed recently for the accurate calculation of the Hartree and the non-local exchange operators have been applied successfully to the $a b$ initio numerical solution of the Hartree-Fock equation for some molecules. In the present work, we show that the rank-structured representation can be gainfully applied to the accurate approximation of the electron density of large Aluminium clusters. We consider the Tucker-type decomposition of the electron density of certain Aluminium clusters originating from finite element calculations in the framework of the orbital-free density functional theory. Numerical investigations of the Tucker approximation of the corresponding electron density reveal the exponential decay of the approximation error with respect to the Tucker rank. The resulting low-rank tensor representation reduces dramatically the storage needs and the computational complexity of the consequent tensor operations on the electron density. As main result, the rank of the Tucker approximation for the accurate representation of the electron density is small and only weakly dependent on the system size for the systems studied here. This shows good promise for resolving the electronic structure of materials using tensor-structured techniques.
\end{abstract}

Keywords: orbital-free density functional theory, Tucker-type

\footnotetext{
*Corresponding author

URL: blesgen@mis.mpg.de (T. Blesgen), vikramg@umich.edu (V. Gavini), vekh@mis.mpg.de (V. Khoromskaia)
} 
decomposition, multigrid accelerated tensor approximation, electron density, Aluminium clusters, finite elements PACS: 71.15.Ap, 31.15.E-, 31.15.xt, 02.60-x 2000 MSC: 81-08, 65Y20, 68P05, 15A23

\section{Introduction}

In this article we are concerned with the accurate and efficient representation of the electronic structure of large, non-periodic systems with hundreds or thousands of atoms. Density functional theory (DFT) as formulated by Hohenberg, Kohn and Sham $[12,20]$ is widely used to compute the ground state properties and electronic structure for a wide range of materials. Although the ground state properties of any material system are only a function of the electron density, the functional representation of the kinetic energy of non-interacting electrons is not known explicitly in terms of the electron density. This makes a direct computation of ground state properties in terms of the electron density inaccessible. This problem is circumvented with the Kohn-Sham approach (KSDFT) where the ground state properties are computed by solving for the wave-functions of a non-interacting system of electrons in a mean field in a self-consistent manner. The complexity associated with the computation of many wavefunctions in a self-consistent manner in the Kohn-Sham approach restricts the size of material systems in most cases to a few hundred atoms. Recently, there have been many efforts towards developing explicit functional representations for the approximate kinetic energy of non-interacting electrons in terms of the electron density $[28,29]$. These approximations which do not require the computations of wavefunctions (or orbitals) constitute the orbital-free density functional theory (OFDFT). The reduced computational complexity of orbital-free densityfunctional theory makes the computation of the electronic-structure of larger material systems possible that are not otherwise accessible by the Kohn-Sham approach to density-functional theory. We note that orbital-free approximations to density functional theory are well-developed for material systems with an electronic structure close to a free-electron gas (simple metals, Aluminium, etc.), but their accuracy in material systems with ionic or covalent bonding is still not satisfactory and this is an active area of research.

In the past, most density functional theory calculations have either used plane-wave basis functions (for solid-state calculations, cf., e. g., [22]) or 
atom-centered atomic orbitals or Gaussian type basis functions (for nonperiodic systems, cf., e. g., [11]). More recently, finite elements are becoming a popular basis set for the computation of the electronic-structure of materials, especially in non-periodic systems $[8,9,24,26]$. The adaptive nature of the basis set, the ease of considering complex geometries and boundary conditions, the local nature of the basis that allows for an efficient parallel implementation are some of the attractive features of finite elements. Although finite elements are better suited for calculations on non-periodic systems in comparison to other commonly-used basis sets like plane-waves or Gaussian basis functions, the number of finite elements required to achieve chemical accuracy $(0.1 \mathrm{eV} /$ atom $)$ is quite large. The size of material systems accessible by a finite element basis where all atoms are accounted for is a few thousand atoms in OFDFT and on the order of a hundred atoms in KSDFT $[8,26]$. However, many interesting material properties, especially those involving defects, occur in much larger systems with millions of atoms which poses a big challenge in materials science. To this end there is a great need to develop appropriate representations for the electronic structure that reduce the computational complexity and the limitations on the accessible sizes of material systems.

Low-rank tensor representations of the multi-dimensional data based on the canonical and Tucker models [27] have been initially considered in signal processing and computer science, see $[4,5,21]$ and references therein. Recent tensor-structured methods $[17,17,10,16,18,19,14]$ reduce dramatically the complexity of the numerical evaluation of the multivariate functions and operators in $\mathbb{R}^{d}, d \geq 3$. Note that conventional numerical methods like the classical finite element method (FEM) have limitations due to their exponential scaling in dimension $d$. The algebraically separable tensor approach proved to be particularly useful for the numerical treatment of 3D problems in electronic structure calculations. In this regard we mention tensor-structured algorithms for the calculation of the Hartree and non-local exchange operators in the Hartree-Fock equation [18, 14].

The appealing perspective of the tensor-structured methods lies in the possibility of the grid-based evaluation of the integral operators in $\mathbb{R}^{d}, d \geq 3$, with linear scaling in the one-dimension grid size $n$, see $[14,15,18]$. In terms of conventional estimation for $3 \mathrm{D}$ problems by volume size $\mathcal{N}_{\text {vol }}=n^{3}$, the tensor-structured operations are of sublinear complexity $O\left(\mathcal{N}_{\text {vol }}^{1 / 3}\right)$.

The present paper is a first step towards applying the tensor-structured algorithms for the OFDFT model. We consider the Tucker approximation to 
the electron density of the Aluminium atomic clusters originating from the finite element calculations in the framework of OFDFT [8]. After interpolating the results of the finite element simulations onto the fine enough uniform 3D Cartesian grid, the multigrid accelerated Tucker decomposition method [18] is applied to find the algebraically separable tensor-product approximation of the original 3D tensor as a sum of rank-1 elementary tensors. These tensors can be used as the basis functions in the same way as the discretized Gaussians are used in $[18,14]$ for the tensor-structured evaluation of the multidimensional convolution integrals. One of the goals of the present paper is to show the existence of the algebraically separable basis of a sufficiently small rank for the electron density of large Aluminium clusters.

Our numerical experiments demonstrate the exponential decay of the Tucker decomposition error of the electron density with respect to the Tucker rank. Hence, the initial 3D tensor representing the electron density of an Aluminium cluster on a $n \times n \times n$ grid with sufficiently large $n$ can be approximated in a separable tensor format using a small number $r^{3}$ (with $r \ll n$ ) of basis functions built from the respective Tucker orthogonal matrices. The storage cost of this representation is $\left(r^{3}+3 r n\right) \ll n^{3}$. Our numerical examples show that the accuracy of the FE approximation of the considered Aluminium clusters can be achieved with rather small Tucker rank; $r \sim 10$ for most test cases considered. The orthogonal vectors of the Tucker decomposition could then be used as the problem-adaptive global basis functions instead of the finite elements.

The results of this investigation suggest that the Tucker rank $r$ is only weakly dependent on the size of Aluminum cluster when varying sizes of the Aluminum clusters with repeating face-centered cubic (FCC) unit cells$1 \times 1 \times 1$ FCC unit cell to $5 \times 5 \times 5$ FCC unit cells - are analyzed. Further, we observe that a small Tucker rank $r \sim 10$ is sufficient for approximating the electron density of these systems. This can be explained by the fact that $r$ characterizes the smoothness properties of the represented function [17] (here the electron density), independent of the size of the discrete system. As more stringent tests, we further considered a system comprising of a single vacancy in a $5 \times 5 \times 5$ Aluminum cluster, as well as a system comprising of 10 vacancies and 10 Lithium substitutional atoms placed at random in a $5 \times 5 \times 5$ Aluminum cluster. While the required rank of the Tucker approximation increased with system complexity, a Tucker rank of $r=20$ is still observed to be sufficient for these more complex systems. We remark that the Tucker rank increases with increasing perturbations of periodic systems, and has 
been reported in previous studies $[14,17]$.

This article is organised in the following way. We begin in Section 2 by the presentation of a real-space formulation of the orbital free density functional theory and discuss the finite element discretization (we refer to [8] for a more comprehensive description). In Section 3 we recall a brief description of the canonical and Tucker-type approximations and the algorithm for the numerical Tucker decomposition [4, 17] and mention some features of the tensor-structured methods. In Section 4 we apply the Tucker model to data from finite element simulations of Aluminium clusters using OFDFT. We observe exponential convergence of the Tucker-type approximation with respect to the Tucker rank and find a dramatic reduction of the required storage for approximating the electron density. In addition we find that the absolute approximation error of the respective decomposition is in the range of the initial accuracy of the finite element scheme for the considered Aluminium clusters. We end with a critical discussion and an outlook.

\section{Orbital-free density functional theory: Real-space formulation and finite element discretization}

Let $N$ denote the number of electrons and $M$ the number of atoms in a charge neutral system. The ground state energy in DFT is given by (see, e.g., $[7,23])$

$$
E(\varrho, \mathbf{R})=T_{s}(\varrho)+E_{\mathrm{xc}}(\varrho)+E_{\mathrm{H}}(\varrho)+E_{\mathrm{ext}}(\varrho, \mathbf{R})+E_{\mathrm{zz}}(\mathbf{R}),
$$

where $\varrho$ denotes the electron density such that

$$
\int_{\Omega} \varrho(\mathbf{x}) \mathrm{d} \mathbf{x}=N
$$

and $\mathbf{R}=\left[\mathbf{R}_{1}, \ldots, \mathbf{R}_{M}\right]$ is the position vector of the nuclei. The term $T_{s}$ denotes the kinetic energy of non-interacting electrons. A typical ansatz in OFDFT is the Thomas-Fermi-von Weizsäcker (TFW) functional, given by

$$
T_{s}(\varrho)=C_{F} \int_{\Omega} \varrho^{5 / 3}(\mathbf{x}) \mathrm{d} \mathbf{x}+\frac{\lambda}{8} \int_{\Omega} \frac{|\nabla \varrho(\mathbf{x})|^{2}}{\varrho(\mathbf{x})} \mathrm{d} \mathbf{x}
$$

for a constant $C_{F}=\frac{3}{10}\left(3 \pi^{2}\right)^{2 / 3}$, a parameter $\lambda>0$, and $\Omega$ contains the compact support of $\varrho$. We note that non-local corrections to the TFW functional have also been proposed and we refer to [28, 29] for more details. 
We also note that numerical investigations over the past decade have shown that orbital-free approximations of the kinetic energy are sufficiently accurate for material systems whose electronic structure is close to free electron gas, e. g. simple metals and Aluminium. However, these approximations fail to produce reasonable accuracy when the bonding is covalent or ionic.

The functional $E_{\mathrm{xc}}$ represents the exchange and correlation energy. Within the framework of local density approximation (LDA), [2], [25], it is given by

$$
E_{\mathrm{xc}}(\varrho)=\int_{\Omega} \varepsilon_{\mathrm{xc}}(\varrho(\mathbf{x})) \varrho(\mathbf{x}) \mathrm{d} \mathbf{x},
$$

and the exchange and correlation energy per electron is defined by

$$
\begin{aligned}
& \varepsilon_{\mathrm{x}}(\varrho)=-\frac{3}{4}\left(\frac{3}{\pi}\right)^{1 / 3} \varrho^{1 / 3}, \\
& \varepsilon_{\mathrm{c}}(\varrho)= \begin{cases}\frac{\gamma}{1+\beta_{1} \sqrt{r_{s}}+\beta_{2} r_{s}} & \text { if } r_{s} \geq 1, \\
A \ln r_{s}+B+C r_{s} \ln r_{s}+D r_{s} & \text { if } r_{s}<1,\end{cases}
\end{aligned}
$$

for $r_{s}=\left(\frac{3}{4 \pi \varrho}\right)^{1 / 3}$ and $\gamma, \beta_{1}, \beta_{2}, A, B, C$ and $D$ are constants. We note that these constants are not material dependent, with the exception that their values are slightly different for materials which are unpolarised and polarised.

For the complete definition of equation (1), we still need to introduce the electrostatic interactions. $E_{\mathrm{H}}$ is the Hartree energy or the electrostatic interaction energy between the electrons, $E_{\text {ext }}$ is the electrostatic interaction energy between electrons and nuclei, and $E_{\mathrm{zz}}$ is the repulsive nucleus-nucleus interaction energy. They are given by

$$
\begin{aligned}
E_{\mathrm{H}}(\varrho) & =\frac{1}{2} \int_{\Omega} \int_{\Omega} \frac{\varrho(\mathbf{x}) \varrho(\mathbf{y})}{|\mathbf{x}-\mathbf{y}|} \mathrm{d} \mathbf{x} \mathrm{d} \mathbf{y}, \\
E_{\mathrm{ext}}(\varrho, \mathbf{R}) & =\sum_{I=1}^{M} \int_{\Omega} \frac{Z_{I}}{\left|\mathbf{x}-R_{I}\right|} \varrho(\mathbf{x}) \mathrm{d} \mathbf{x}, \\
E_{\mathrm{zz}}(\mathbf{R}) & =\frac{1}{2} \sum_{I=1}^{M} \sum_{J=1, J \neq I}^{M} \frac{Z_{I} Z_{J}}{\left|R_{I}-R_{J}\right|},
\end{aligned}
$$

where $Z_{I}<0$ is the value of the nuclear charge of the $I$-th atom. We note that it is conventional to treat electron density to be positive and nuclear charges to be negative in electronic structure calculations. 
We remark that, in the framework of orbital-free DFT using TFW kinetic energy functionals, the various energy components are local except for the electrostatic interactions. However, we note that the electrostatic interactions are convolutions with a $\frac{1}{|\mathbf{x}|}$ kernel, which is the Green's function for the Laplace operator. With this observation, the electrostatic interaction energy can be reformulated in a local form as the following variational problem:

$$
\begin{aligned}
& \frac{1}{2} \int_{\Omega} \int_{\Omega} \frac{\varrho(\mathbf{x}) \varrho(\mathbf{y})}{|\mathbf{x}-\mathbf{y}|} d \mathbf{x} d \mathbf{y}+\int_{\Omega} \int_{\Omega} \frac{\varrho(\mathbf{x}) b(\mathbf{y} ; \mathbf{R})}{|\mathbf{x}-\mathbf{y}|} d \mathbf{x} d \mathbf{y}+\frac{1}{2} \int_{\Omega} \int_{\Omega} \frac{b(\mathbf{x} ; \mathbf{R}) b(\mathbf{y} ; \mathbf{R})}{|\mathbf{x}-\mathbf{y}|} d \mathbf{x} d \mathbf{y} \\
& =-\inf _{\phi \in H_{0}^{1}(\Omega)}\left\{\frac{1}{8 \pi} \int|\nabla \phi(\mathbf{x})|^{2} d \mathbf{x}-\int(\varrho(\mathbf{x})+b(\mathbf{x} ; \mathbf{R})) \phi(\mathbf{x}) d \mathbf{x}\right\}
\end{aligned}
$$

where $\phi$ denotes a trial function for the electrostatic potential of the system of charges, and $b(\mathbf{x} ; \mathbf{R})=\sum_{I} Z_{I} \delta\left(\mathbf{x}-R_{I}\right)$ with $\delta\left(\mathbf{x}-R_{I}\right)$ representing the regularized nuclear charges, which, in pseudopotential calculations, represents a nuclear charge distribution corresponding to a pseudopotential. We remark that the left hand side of equation (2) differs from the sum of electrostatic terms by the self energy of the nuclei, which is an inconsequential constant that goes along with the choice of the pseudopotential.

The ground state electron density is determined by minimizing the energy with respect to the electron density $\varrho$ under the constraints $\varrho \geq 0$ and $\int_{\Omega} \varrho=$ $N$. The constraint $\varrho \geq 0$ is introduced through the substitution $\varrho=u^{2}$, and the problem of determining the ground state electron density and energy for fixed positions of nuclei is given by the saddle point problem

$$
\min _{u \in H_{0}^{1}(\Omega)} \max _{\phi \in H_{0}^{1}(\Omega)} L(\phi, u, \mathbf{R}) \quad \text { subject to } \int_{\Omega} u^{2} \mathrm{~d} \mathbf{x}=N,
$$

where the Lagrangian $L(\phi, u, \mathbf{R})$ is given by

$$
\begin{aligned}
L(\phi, u, \mathbf{R})= & \frac{\lambda}{2} \int_{\Omega}|\nabla u(\mathbf{x})|^{2} \mathrm{~d} \mathbf{x}-\frac{1}{8 \pi} \int_{\Omega}|\nabla \phi(\mathbf{x})|^{2} \mathrm{~d} \mathbf{x}+C_{F} \int_{\Omega} u^{10 / 3}(\mathbf{x}) \mathrm{d} \mathbf{x} \\
& +\int_{\Omega} u^{2}(\mathbf{x}) \varepsilon_{\mathbf{x c}}\left(u^{2}(\mathbf{x})\right) \mathrm{d} \mathbf{x}+\int_{\Omega}\left(u^{2}(\mathbf{x})+b(\mathbf{x} ; \mathbf{R})\right) \phi(\mathbf{x}) \mathrm{d} \mathbf{x} .
\end{aligned}
$$

The equation (3) describes the ground state properties of a material system in the OFDFT framework as a local variational formulation in real-space. 
Thus, a finite element basis set which respects this local structure is a natural choice for the discretization. Further, finite element basis functions allow for arbitrary boundary conditions and consideration of complex geometries, unlike more structured basis functions like plane-waves. The local nature of the finite elements also aids in an efficient parallel implementation of the calculation, which is especially important for electronic structure calculations that are computationally demanding, both in terms of computing cycles as well as memory requirements. If $T_{h}$ denotes the finite element triangulation of the domain $\Omega$, and $X_{h}$ denotes the subspace spanned by the corresponding finite element basis functions that becomes increasingly dense in $H_{0}^{1}(\Omega)$, the saddle point problem given by equation (3) reduces to a constrained saddle point problem given by

$$
\inf _{u^{h} \in X_{h}} \sup _{\phi^{h} \in X_{h}} L\left(\phi^{h}, u^{h}, \mathbf{R}\right) \quad \text { subject to } \int\left(u^{h}(\mathbf{x})\right)^{2} \mathrm{~d} \mathbf{x}=N .
$$

We refer to [8] for a comprehensive discussion on the mathematical properties of the finite element discretization in equation (4), the numerical implementation of the method, and computations of the electronic structure of Aluminium clusters of varying sizes - from 14 atoms to a few thousand atoms.

\section{Rank-structured representation of the full-size tensors}

This section recalls the general ideas and some basic definitions on the tensor product algorithms given in $[4,5,21]$ which have been developed further towards the tensor-structured methods for the problems of numerical analysis in higher dimensions $[17,18,19,14]$. The numerical examples considered in the latter references include, in particular, the efficient 3D tensor-product convolution with the Newton kernel and accurate numerical evaluation of the non-local integral operators in electronic structure calculations.

A real tensor of order $d$ is a multidimensional array whose elements are referred to by an index set $\mathcal{I}=I_{1} \times \ldots \times I_{d}$,

$$
A=\left[a_{i_{1} \ldots i_{d}}: i_{\ell} \in I_{\ell}\right] \in \mathbb{R}^{\mathcal{I}}, \quad I_{\ell}=\left\{1, \ldots, n_{\ell}\right\}, \ell=1, \ldots, d .
$$

The tensor $A$ is an element of the tensor-product linear space $\mathbb{V}_{\mathbf{n}}=\otimes_{\ell=1}^{d} \mathbb{V}_{\ell}$ with $\mathbb{V}_{\ell}=\mathbb{R}^{I_{\ell}}$, equipped with the Euclidean inner product $\langle\cdot, \cdot\rangle: \mathbb{V}_{\mathbf{n}} \times \mathbb{V}_{\mathbf{n}} \rightarrow$ 
$\mathbb{R}$, defined as

$$
\langle A, B\rangle:=\sum_{\left(i_{1}, \ldots, i_{d}\right) \in \mathcal{I}} a_{i_{1} \ldots i_{d}} b_{i_{1} \ldots i_{d}} \quad \text { for } A, B \in \mathbb{V}_{\mathbf{n}}
$$

The related Euclidean (Frobenius) norm is

$$
\|A\|_{F}:=\sqrt{\langle A, A\rangle}
$$

The number of entries in $A$ is $S(A):=\prod_{\ell=1}^{d} n_{\ell}$. Hence, assuming $n_{\ell}=n$, we obtain $S(A)=n^{d}$. To get rid of the exponential scaling in $d$ we use the "rank-structured" separable approximation of tensors.

The simplest example of rank-structured tensors is the canonical rank-1 tensor which is formed by the tensor product of $d \ell$-mode vectors $u^{(\ell)}=$ $\left\{u_{i_{\ell}}^{(\ell)}\right\}_{i_{\ell} \in I_{\ell}} \in \mathbb{V}_{\ell}(\ell=1, \ldots, d)$,

$$
U \equiv\left[u_{\mathbf{i}}\right]_{\mathbf{i} \in \mathcal{I}}=u^{(1)} \otimes \ldots \otimes u^{(d)} \in \mathbb{V}_{\mathbf{n}} \quad \text { with entries } \quad u_{\mathbf{i}}=u_{i_{1}}^{(1)} \cdots u_{i_{d}}^{(d)} .
$$

This tensor requires only $d n$ numbers to store (now linear scaling in $d$ ). For $d=2$, the tensor product of two vectors represents a rank-1 matrix. A generic tensor cannot be represented by a canonical rank-1 tensor with a given accuracy, hence we are enforced to use more appropriate constructions.

For the ease of presentation, in the following, we consider only the 3D tensors, $A=\left[a_{i_{1} i_{2} i_{3}}\right], i_{\ell} \in I_{\ell}$ for $\ell=1,2,3$.

A set of tensors represented by a sum of rank- 1 tensors with a given rank parameter $R \in \mathbb{N}$ is called a canonical format of tensors,

$$
A_{(R)}=\sum_{\nu=1}^{R} c_{\nu} u_{\nu}^{(1)} \otimes u_{\nu}^{(2)} \otimes u_{\nu}^{(3)}, \quad c_{\nu} \in \mathbb{R},
$$

where $u_{\nu}^{(\ell)} \in \mathbb{V}_{\ell}(\ell=1,2,3)$ are normalised vectors. The minimal parameter $R$ in (6) is called the (canonical) rank of a tensor. The storage cost is bounded by $3 R n$ with $n=\max _{\ell}\left\{n_{\ell}\right\}$.

The canonical representation is useful in multilinear tensor operations. In [17] it is shown that the bi-linear operations with tensors in the rank- $R$ canonical format have linear complexity $O(n)$ with respect to the univariate grid size $n$ of the function-generated tensor. The disadvantage of this presentation consists in the absence of fast and stable algorithms for transforming 
the arbitrary full-size tensors into the canonical format with the controlled accuracy.

The Tucker tensor model provides robust algorithms for the decomposition of full-size tensors into the rank-structured format. The rank- $\left(r_{1}, r_{2}, r_{3}\right)$ Tucker representation (approximation) for a given 3rd order tensor $A=$ $\left[a_{i_{1} i_{2} i_{3}}\right], i_{\ell} \in I_{\ell}(\ell=1,2,3)$ is defined as

$$
A_{(\mathbf{r})}:=\sum_{\nu_{1}=1}^{r_{1}} \sum_{\nu_{2}=1}^{r_{2}} \sum_{\nu_{3}=1}^{r_{3}} \beta_{\nu_{1} \nu_{2} \nu_{3}} v_{\nu_{1}}^{(1)} \otimes v_{\nu_{2}}^{(2)} \otimes v_{\nu_{3}}^{(3)} .
$$

The vectors $v_{\nu_{\ell}}^{(\ell)} \in \mathbb{R}^{n_{\ell}}\left(1 \leq \nu_{\ell} \leq r_{\ell}\right)$ form an orthonormal basis of $\mathbb{T}_{\ell}=$ $\operatorname{span}_{1 \leq \nu_{\ell} \leq r_{\ell}}\left\{v_{\nu_{\ell}}^{(\ell)}\right\} \quad(\ell=1,2,3)$ with $\operatorname{dim} \mathbb{T}_{\ell}=r_{\ell}$. Here, $r:=\max _{\ell}\left\{r_{\ell}\right\}$ is called the Tucker rank. The coefficients tensor $\boldsymbol{\beta}=\left[\beta_{\nu_{1} \nu_{2} \nu_{3}}\right]$, an element of the tensor space $\mathbb{B}_{\mathbf{r}}:=\mathbb{R}^{r_{1} \times r_{2} \times r_{3}}, \mathbf{r}:=\left(r_{1}, r_{2}, r_{3}\right)$, is called the core tensor. Since the storage complexity of the Tucker representation (7) is bounded by $r^{3}+3 r n$, it takes the advantage if $r \ll n$, which is usually the case in our applications.

Using the orthogonal side matrices $V^{(\ell)}=\left[v_{1}^{(\ell)} \ldots v_{r_{\ell}}^{(\ell)}\right]$, we represent the Tucker decomposition of $A$ using the contracted products,

$$
A_{(\mathbf{r})}=\boldsymbol{\beta} \times{ }_{1} V^{(1)} \times_{2} V^{(2)} \times{ }_{3} V^{(3)},
$$

where the $\ell$-mode contracted product $x_{\ell}$ involves the summation over the $\ell$-th dimension, $\ell=1,2,3$ (see Appendix).

Figure 1 shows the orthogonal $\ell$-mode side matrices and the core tensor of the Tucker tensor decomposition for the tensor with $d=3$. It was shown, [17], that the Tucker approximation error for a class of 3D function-related tensors decays exponentially with respect to the Tucker rank, that the Tucker rank $r$ only weakly depends on $n$, and the shape of the orthonormal vectors in the side matrices characterizes the discretized function and does not depend on $n$.

A rank- $\left(r_{1}, \ldots, r_{d}\right)$ Tucker approximation can be formulated as the solution of a minimisation problem: For a given initial tensor $A_{0} \in \mathbb{V}_{\mathbf{n}}$ compute the optimal $A$ in the orthogonal Tucker format,

$$
f(A):=\left\|A-A_{0}\right\|_{F}^{2} \rightarrow \min \quad \text { over } A \in \mathcal{T}_{\mathbf{r}} .
$$

The minimisation problem (9) is equivalent to the maximisation [5]

$$
g\left(V^{(1)}, \ldots, V^{(d)}\right):=\left\|A_{0} \times_{1}\left(V^{(1)}\right)^{T} \times \ldots \times_{d}\left(V^{(d)}\right)^{T}\right\|^{2} \rightarrow \max
$$




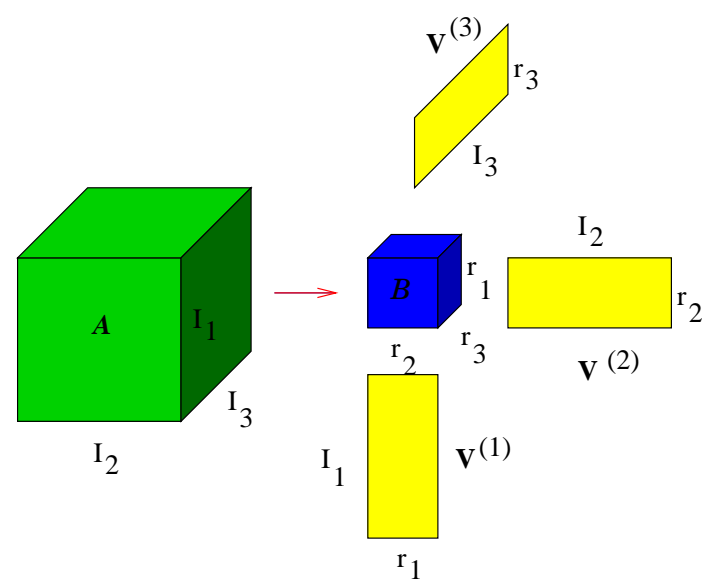

Figure 1: Tucker tensor decomposition for the tensor with $d=3$.

over the set of orthogonal matrices $V^{(\ell)} \in \mathbb{R}^{n_{\ell} \times r_{\ell}}, \ell=1, \ldots, d$.

In the case $d=3$, for given matrices $V^{(\ell)}$, the core tensor $\boldsymbol{\beta}$ that minimises (9) is represented by

$$
\boldsymbol{\beta}=A_{0} \times_{1}\left(V^{(1)}\right)^{T} \times_{2}\left(V^{(2)}\right)^{T} \times_{3}\left(V^{(3)}\right)^{T} \in \mathbb{R}^{r_{1} \times r_{2} \times r_{3}} .
$$

The algorithm for the best Tucker approximation based on the alternating least squares (ALS) procedure is proposed in [4].

Below we present the main steps of the best Tucker approximation (BTA) algorithm for the decomposition of the full size tensor $A_{0} \in \mathbb{V}_{\mathbf{n}}$ to the Tucker format. It is given for the case $d=3$ for simplicity of presentation.

1. Compute the initial guess $V_{0}^{(\ell)}(\ell=1,2,3)$ for the $\ell$-mode sidematrices by the truncated singular value decomposition (SVD) applied to matrix unfolding $A_{(\ell)}$ (see Appendix). This step is of the largest complexity $O\left(n^{4}\right)$.

2. For $k=1: k_{\max }$ do: for each $q=1,2,3$, and with fixed sidematrices $V^{(\ell)} \in \mathbb{R}^{n \times r_{\ell}}, \ell \neq q$, optimise the side matrix $V^{(q)}$ by computing the dominating $r_{q}$-dimensional subspace. It is computed by the truncated SVD of the respective (reduced) matrix unfolding $B_{(q)} \in \mathbb{R}^{n_{q} \times \bar{r}_{q}}, \bar{r}_{q}=\prod_{\ell=1, \ell \neq q}^{3} r_{\ell}$. The corresponding $q$-mode contracted products are (see Appendix)

$$
\begin{aligned}
& B_{(1)}=A_{0} \times_{2}\left(V^{(2)}\right)^{T} \times_{3}\left(V^{(3)}\right)^{T}, \\
& B_{(2)}=A_{0} \times_{1}\left(V^{(1)}\right)^{T} \times_{3}\left(V^{(3)}\right)^{T}, \\
& B_{(3)}=A_{0} \times_{1}\left(V^{(1)}\right)^{T} \times_{2}\left(V^{(2)}\right)^{T} .
\end{aligned}
$$


For function related tensors the Tucker decomposition needs only a few ALS iterations, for example, $k_{\max }=3$.

3. Compute the core $\boldsymbol{\beta}$ as the representation coefficients of the orthogonal projection of $A_{0}$ onto $\mathbb{T}_{\mathbf{n}}=\otimes_{\ell=1}^{3} \mathbb{T}_{\ell}$ with $\mathbb{T}_{\ell}=\operatorname{span}\left\{v_{\nu_{\ell}}^{(\ell)}\right\}_{\nu_{\ell}=1}^{r_{\ell}}$, by contracted products

$$
\boldsymbol{\beta}=A_{0} \times_{1}\left(V^{(1)}\right)^{T} \times_{2}\left(V^{(2)}\right)^{T} \times_{3}\left(V^{(3)}\right)^{T} .
$$

The complexity of this algorithm scales as $n^{d+1}$, in our case $O\left(n^{4}\right)$. Therefore, the direct Tucker decomposition is computationally unfeasible for the cubic grids of the size larger than $128^{3}$ on a SUN workstation, as we are currently using Matlab for the computations. This limitation can be avoided by using the multigrid accelerated (MGA) Tucker decomposition introduced in [18]. It is based on the successive reiteration of the ALS Tucker approximation on a sequence of refined grids, using the results of the coarse grid approximation as the initial guess for the dominating subspaces on finer grid levels. The resulting complexity of the MGA Tucker decomposition for the full-size tensors is $O\left(n^{d}\right)$, which currently enables application of this algorithm to the $3 \mathrm{D}$ function-related tensors with the maximum size of an $n \times n \times n$ Cartesian grid in the range of $512^{3}$ entries. In fact, for the MGA Tucker decomposition, applications are only limited by the available storage size for the input tensor.

Using the above algorithm, the mapping matrices $V^{(\ell)}$ are calculated to approximate the initial full size tensor $A=\left[a_{i_{1} i_{2} i_{3}}\right]$ (having a pointwise representation (5)) by a separable tensor of type (7), where the core tensor $\boldsymbol{\beta}$ consists of coefficients $\beta_{\nu_{1} \nu_{2} \nu_{3}}$ for the rank-1 tensors

$$
v_{\nu_{1}}^{(1)} \otimes v_{\nu_{2}}^{(2)} \otimes v_{\nu_{3}}^{(3)} .
$$

In this way, we find a separable approximation of the initial tensor with a required accuracy, using $r^{3}$ rank-1 discrete basis functions (12). According to [14] one can easily make the canonical representation (6) of a Tucker tensor using at most $r^{2}$ canonical tensors of rank-1. This representation is useful for further calculation of the $3 \mathrm{D}$ potentials and integral operators in the tensor-structured format [14].

In fact, the numerical integration in $3 \mathrm{D}$ is then reduced to a sequence of $3 \mathrm{D}$ discrete convolutions with the corresponding Hadamard and scalar products, where all operations have linear complexity in the one-dimension grid size $n[15,18]$. For example, for a pair of tensors in the canonical format 
the convolution transform is computed in

$$
O\left(d R_{1} R_{2} n \log n\right)
$$

operations, where $R_{1}$ and $R_{2}$ are the canonical ranks of the respective target tensors. The complexity (13) of the tensor-structured convolution proposed in [15] essentially outperforms the best existing exact $O\left(n^{3} \log n\right)$-methods based on the 3D FFT. The numerics on the tensor-product convolution with the Newton potential in application to the electronic structure calculations is presented in $[18,19,14]$.

\section{Numerical results}

In this section we investigate the accuracy and effectiveness of the Tuckertype decomposition of the electronic structure computed from orbital-free density functional theory. In particular we are interested in understanding the properties of this decomposition for varying system sizes, which will determine the viability of tensor decomposition techniques for large scale electronic structure computations. To this end, we use the real-space formulation of orbital-free density functional theory and a finite element discretization of the formulation, as discussed in Section 2, to compute the ground state electronic density of varying sizes of Aluminium clusters. The different clusters considered in this study are a single Aluminium atom and clusters with $1 \times 1 \times 1,3 \times 3 \times 3,4 \times 4 \times 4$ and $5 \times 5 \times 5$ FCC unit cells. In the subsequent text we denote them by cluster 1 to cluster 5 consisting of $14,172,365$, and 666 Aluminium atoms, respectively. The simulations on the Aluminium clusters are performed using the TFW kinetic energy functional with $\lambda=\frac{1}{6}$, local density approximation for the exchange correlation energy [25] and an evanescent-core pseudopotential for Aluminium [6].

In finite element computations, the computational domain is chosen to be about a hundred times the size of the cluster and Dirichlet boundary conditions are applied on the boundary of this domain as the electronic fields comprising of electron density and electrostatic potential decay away from the cluster. The finite element triangulations, comprising of linear tetrahedral elements that rapidly coarse-grain away from the cluster for computational efficiency, are chosen such that the error in energy is within $0.1 \mathrm{eV} /$ atom (chemical accuracy). We refer to [8] for details on coarse-graining rates for the triangulations and numerical aspects of these computations. 
We begin our analysis by considering the electronic structure of a single Aluminium atom. We compute the electronic structure on two finite element triangulations, a coarse triangulation containing 599, 040 elements and a fine triangulation, obtained from a uniform subdivision of the coarse triangulation using the Freudenthal's tetrahedron subdivision algorithm [1], which has 4, 792, 320 elements.

Next we represent the electron density from the finite element calculations on the 3D Cartesian $n \times n \times n$ grid using the standard Matlab interpolation procedure. In this test case and those to follow, we only use the electron density from the cluster region to assess the efficiency of the Tucker representation. Since the accuracy level for the considered examples of the finite element data was sufficient with $n=250$, there was no need for finer grids. Then the initial tensor $A_{0}$ is transformed by the MGA Tucker decomposition with the rank $\mathbf{r}=\left(r_{1}, r_{2}, r_{3}\right)$ and equal $\ell$-mode ranks $r_{\ell}=r,(\ell=1,2,3)$, where the rank-parameter $r$ increases from $r=1,2, \ldots$ to some predefined value.

The orthogonal vectors and the Tucker core of the size $r \times r \times r$ are then used for the reconstruction of the approximating tensor $A_{(\mathbf{r})} \approx A_{0}$ on the 3D Cartesian grid for estimating the approximation properties of the tensor decomposition with the given rank. For every rank $\mathbf{r}$ Tucker decomposition, we compute the relative error in the Frobenius norm as in [17]

$$
E_{F}=\frac{\left\|A_{0}-A_{(\mathbf{r})}\right\|_{2}}{\left\|A_{0}\right\|_{2}}
$$

and the relative error in the $\ell^{2}$-energy

$$
E_{E N}=\frac{\left|\left\|A_{0}\right\|_{2}-\left\|A_{(\mathbf{r})}\right\|_{2}\right|}{\left\|A_{0}\right\|_{2}} .
$$

Figure 2 shows the convergence of the Tucker approximation in terms of the relative error in the Frobenius norm and $\ell^{2}$-energy norm for the coarse and fine triangulations. Note that there is an exponential drop in the approximation error for small Tucker ranks, and we obtain good convergence with respect to the relative error in Frobenius norm up to $2.4 \cdot 10^{-3}$ with a small Tucker rank $r=4$. However, no significant improvement in the errors is observed beyond $r=5$. We remark that this stagnation of the error is a consequence of the accuracy of the finite element OFDFT data that cannot be surpassed. This is evident from the fact that Tucker approximation errors 

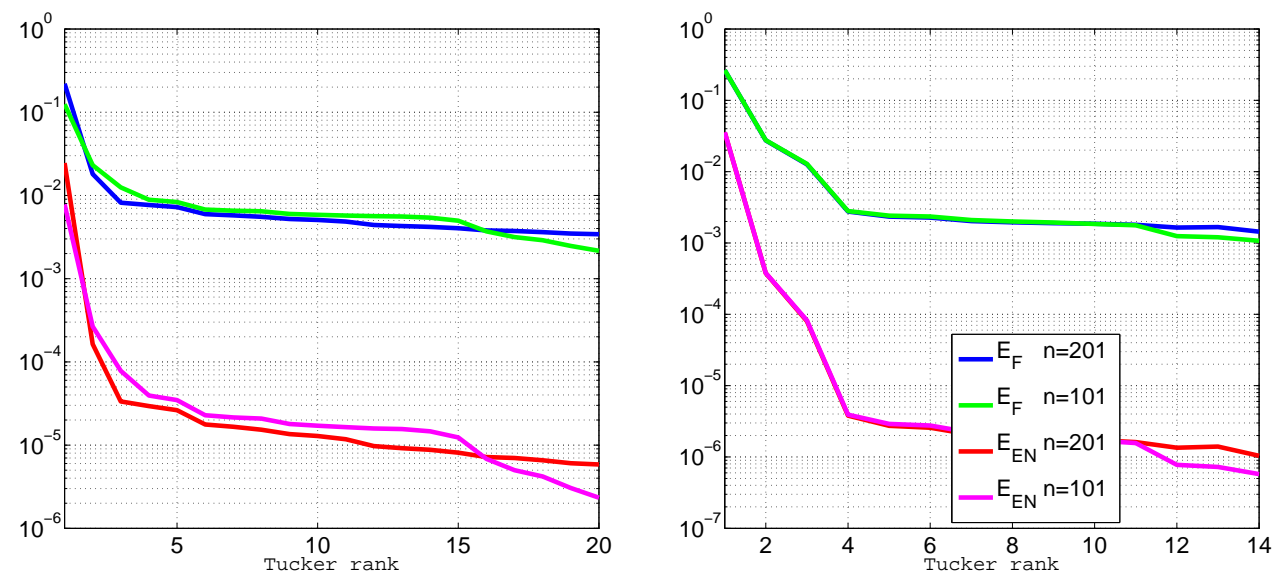

Figure 2: Convergence with respect to the Tucker rank $r$ in Frobenius and energy norms for one Aluminium atom, coarse triangulation (left) and fine triangulation (right).

are almost one order lower in the fine triangulation in comparison to the coarse triangulation.

Next we consider the Tucker approximation of the electron density for cluster 1 to cluster5. The finite element computations are performed on triangulations with 8,646, 656 elements for cluster1, 20,643, 840 elements for cluster3, 39, 456, 768 elements for cluster4, and 57, 716, 736 elements for cluster5. Figure 3 shows the electron density contours along a slice through cluster1 and cluster5. Fig. 4 shows the first 6 basis functions of the Tucker tensor approximation for cluster1 (left) and cluster5 (right), respectively. These vectors constitute one of the orthogonal mapping matrices $V^{(\ell)}, \ell=1,2,3$, obtained by the multilinear singular value decomposition in the Tucker ALS algorithm. As the figures demonstrate, the form of the basis functions depends on the shape of the considered material system, therefore they provide good approximation properties as seen in the subsequent Fig. 5. Fig. 5 presents the absolute error of the Tucker approximation with the rank $r=12$ for cluster5. The maximum absolute errors of the approximation are of the order $5.10^{-4}$ which is in the range of the error bound for the finite element approximation.

Fig. 6 shows the convergence of the relative error in Frobenius norm $E_{F}$ and the relative error in $\ell^{2}$-energy $E_{E N}$ with respect to the Tucker rank $r$ for 

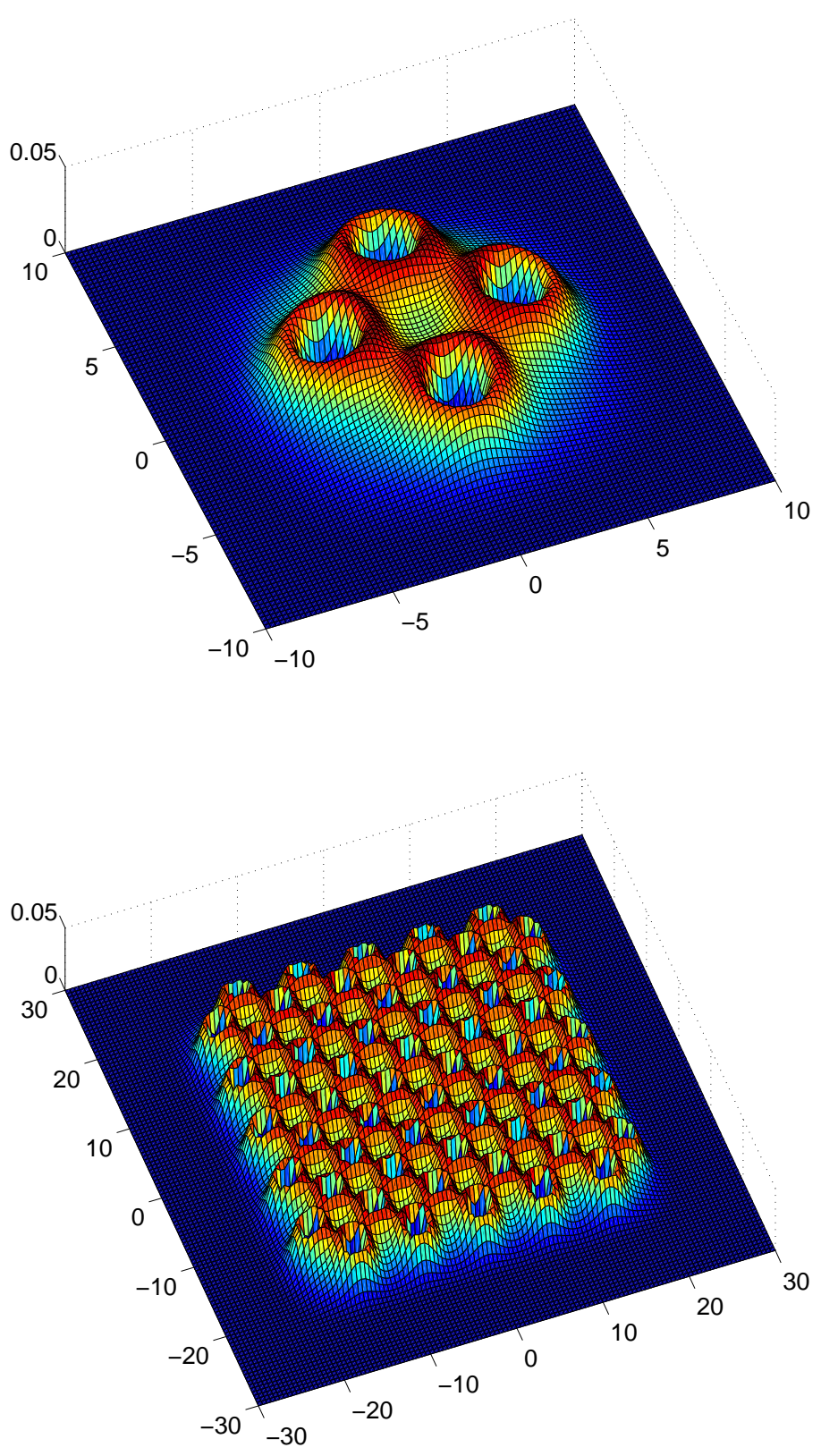

Figure 3: Electron density of Aluminium on the regular grid $n \times n \times n$ for $n=200$, in the subinterval $[-10,10] \times[-10,10] \times\{0\}$ for cluster1 (top), and in the subinterval $[-30,30] \times[-30,30] \times\{0\}$ for cluster5 (bottom). 

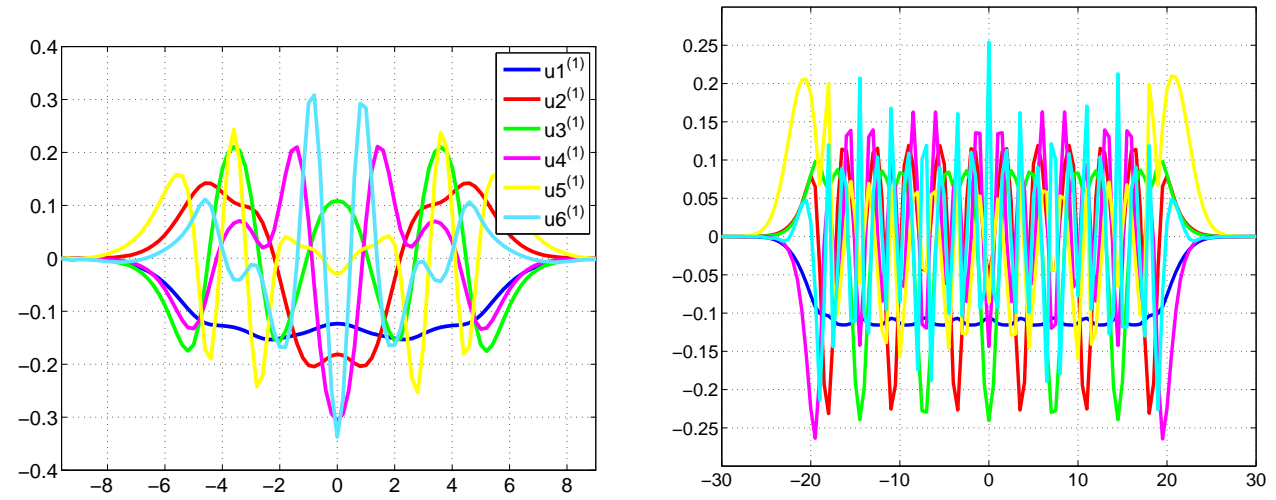

Figure 4: Orthogonal vectors of matrices $T^{(\ell)}$ for $\ell=1$ of the Tucker decomposition with the rank $r=6$ for cluster1 (left) and cluster5 (right).

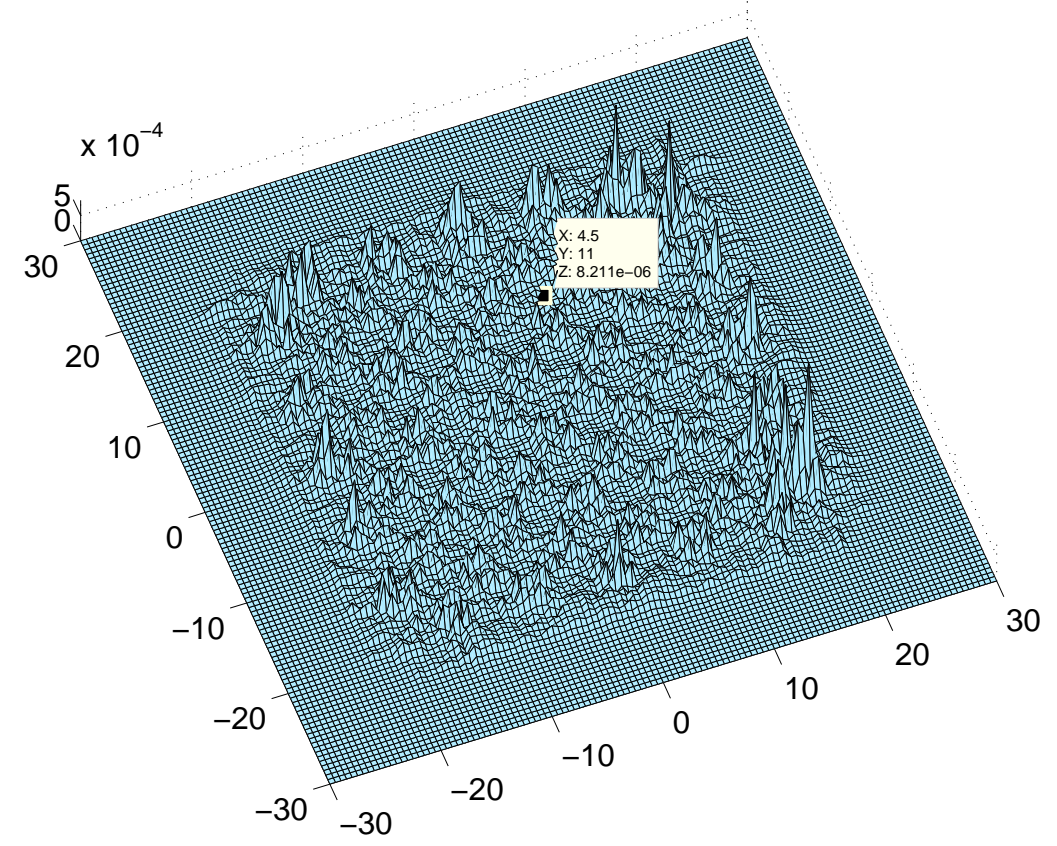

Figure 5: The absolute approximation error of the Tucker representation for cluster5 with $r=12, n=241$ in the subinterval $\Omega=[-30,30] \times[-30,30] \times\{0\}$. 

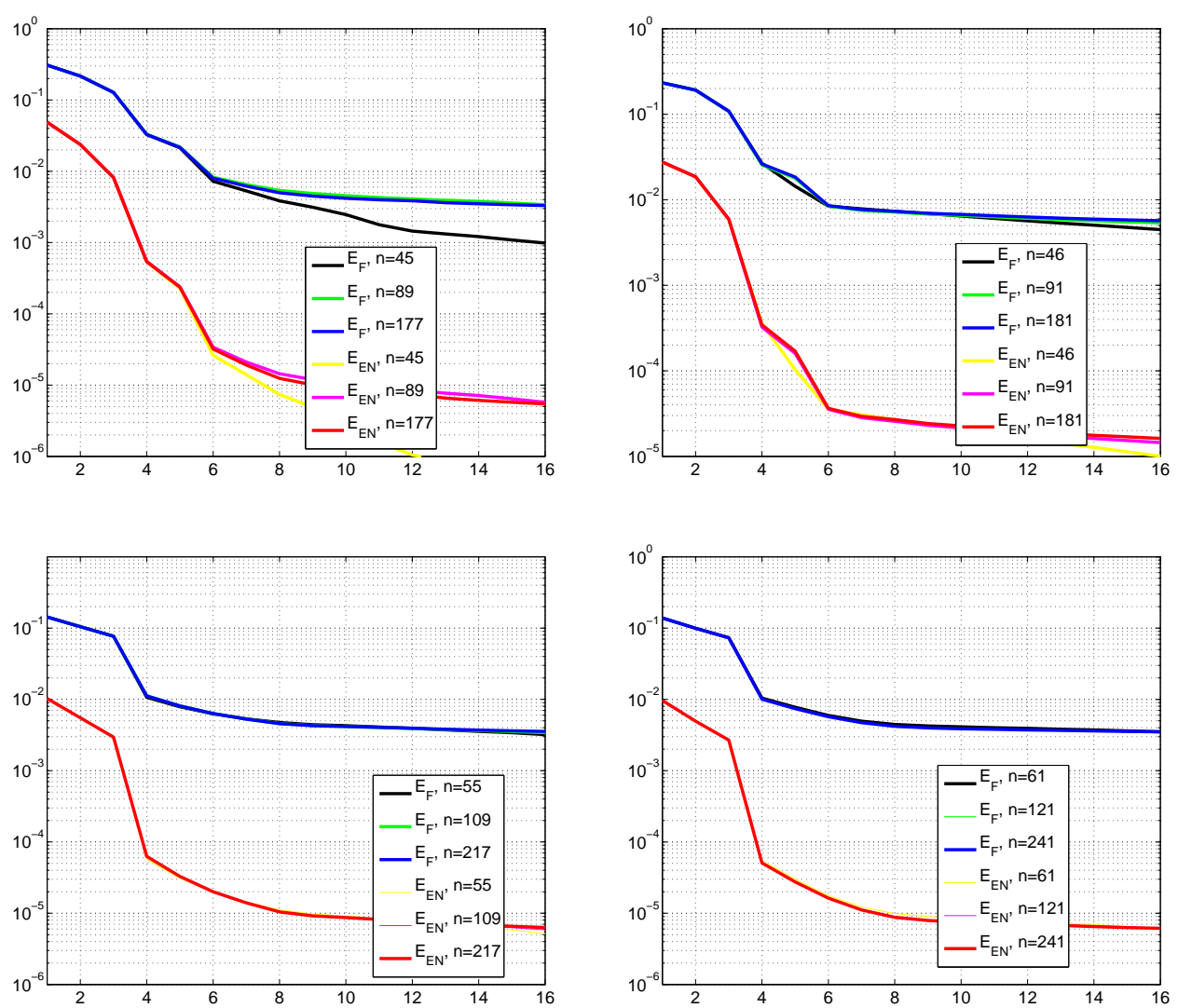

Figure 6: Error convergence of the Tucker tensor approximation with respect to the increasing Tucker rank for cluster1 with 14 atoms (top left), cluster3 with 172 atoms (top right), cluster 4 with 365 atoms (bottom left), and cluster5 with 666 atoms (bottom right) in Frobenius and energy norms. 
the interpolated data on the Cartesian grids. Here we have chosen the univariate grid sizes $n=45,89,177$ for cluster1 with 14 atoms; $n=46,91,181$ for cluster3 with 172 atoms; $n=55,109,217$ for cluster4 with 365 atoms; and $n=61,121,241$ for cluster5 with 666 atoms. Firstly, we note that the convergence is exponential for small Tucker ranks before saturation of these errors occurs resulting from the finite accuracy of the finite element solutions. A relative Frobenius error of less than $10^{-2}$ and a relative $\ell^{2}$-energy error of less than $10^{-5}$ are obtained using just a Tucker rank of $r=10$ for the various clusters considered in this study. Another important feature of this approximation, as seen from the results in Fig. 6, is that the Tucker approximation is only weakly dependent on the univariate grid size as remarked in Section 3.

Next, to further demonstrate the applicability of the Tucker approximation, we consider the Tucker decomposition of cluster5 with an asymmetric vacancy, see Fig. 7, top. Fig. 7 (left bottom) shows the orthonormal vectors of one of the Tucker side matrices $V^{(1)}=\left[v_{1}^{(1)} \ldots v_{r_{1}}^{(1)}\right]$, with $r_{1}=6$. It is clearly seen that the 5 -th orthogonal vector of the side matrix $V^{(1)}$ reveals the shape and location of the vacancy in the Aluminium cluster. Fig. 7 (right bottom) shows the convergence of the approximation error for the cluster5 with a vacancy. We observe only a slight decrease in the convergence of the approximation error as compared with the results for cluster5.

The figures 8 to 9 show the results of computations for the cluster with originally 666 Aluminium atoms where 20 Aluminum atoms have been replaced at random by 10 vacancies and 10 Lithium atoms. The top pictures in Fig. 8 present two sectional planes of this cluster. Two Lithium atoms can be identified in the upper left corner of the plane $z=0$ (left), and two vacancies and two Lithium atoms at the sectional plane $y=-8$ (right). Figure 8, bottom shows the exponential convergence of the approximation error with respect to the Tucker rank. One can notice in Figures 9, left and right, that the orthogonal Tucker vectors $v_{1}^{(2)}, \ldots, v_{7}^{(2)}$ and $v_{1}^{(3)}, \ldots, v_{7}^{(3)}$, respectively, reveal the location of random $\mathrm{Li}$ atoms and vacancies in the Aluminium cluster.

The numerical investigations show that the electronic structure of Aluminium clusters of different sizes and complexity can be captured very efficiently using the Tucker representation as the error convergence is exponential with the Tucker rank. This is similar to spectral convergence using planewave basis functions for electronic structure calculations, but can be achieved now in a non-periodic setting with very few basis functions in comparison 

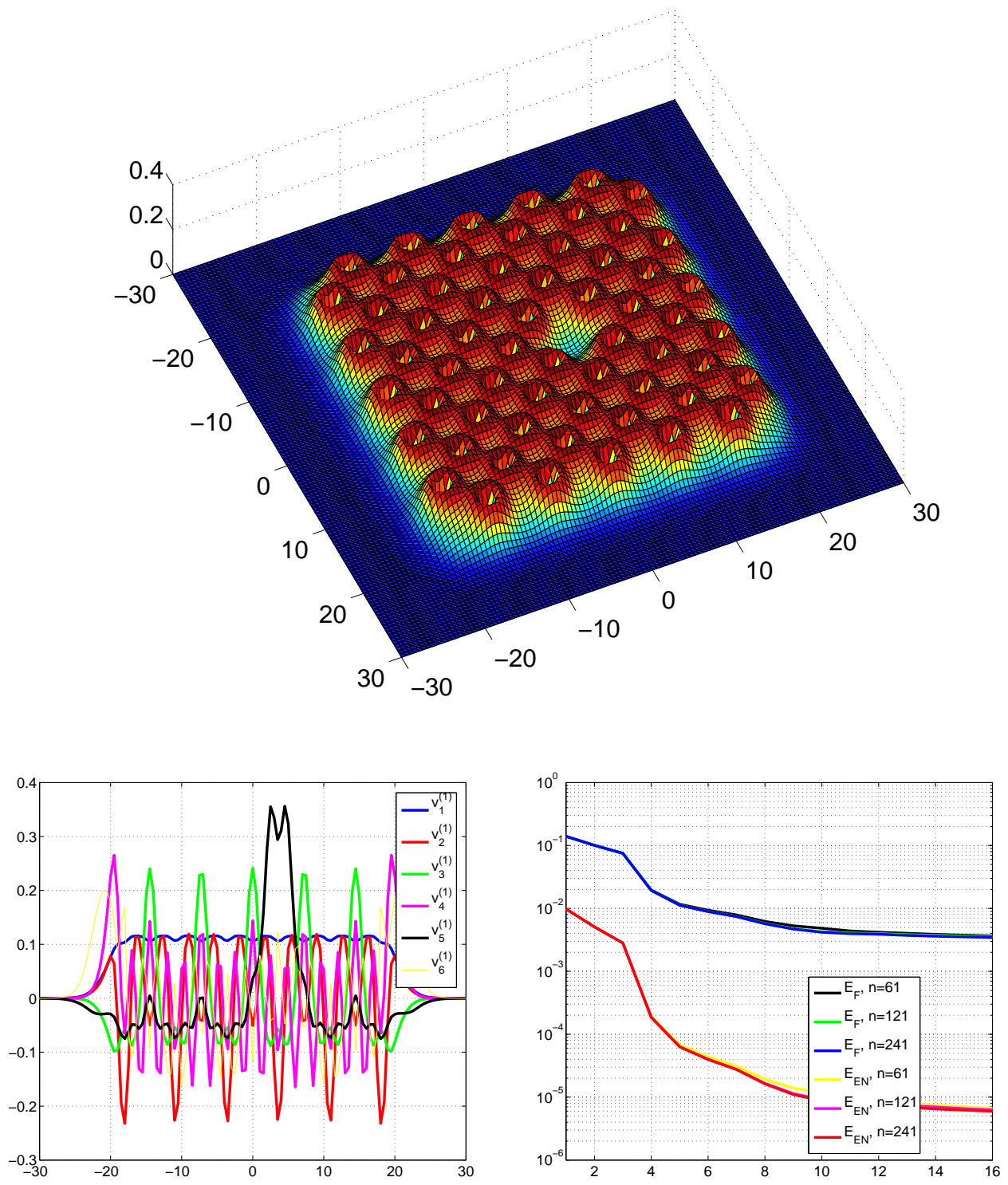

Figure 7: Electron density of an Aluminium cluster with 666 atoms with an asymmetric vacancy (top). The left bottom figure shows the orthogonal Tucker vectors $v_{1}^{(1)}, \ldots, v_{6}^{(1)}$. It is seen that the 5-th vector of the Tucker mode-1 side matrix "reveals" the shape and location of the vacancy. The right bottom figure shows that the convergence of the approximation error is not significantly affected by the presence of a vacancy in the cluster. 

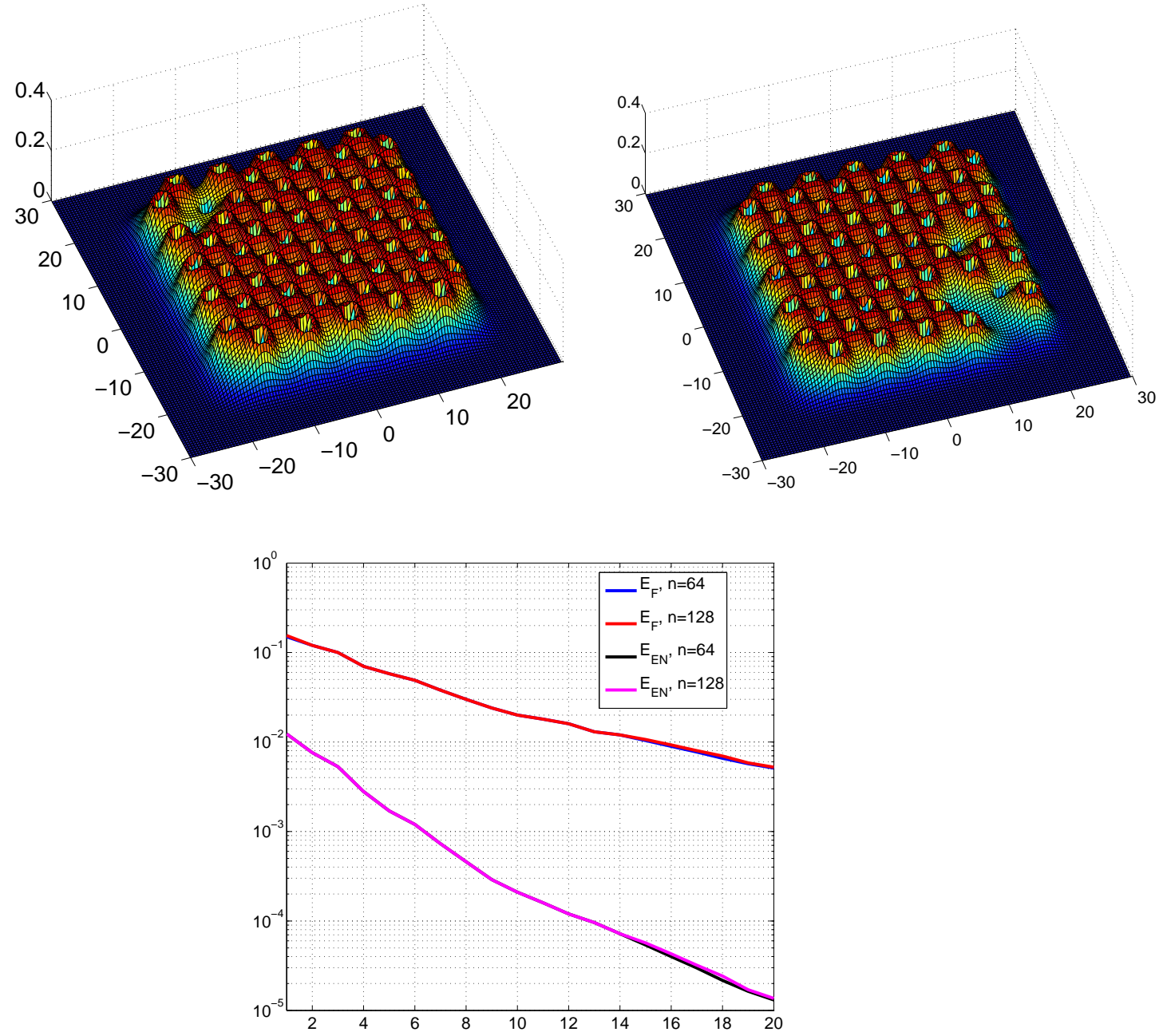

Figure 8: Electron density of a cluster with $666 \mathrm{Al}$ atoms with random replacement of 20 Aluminium atoms by 10 Lithium atoms and 10 vacancies: two sectional planes with $z=0$ (left) and $y=-8$ (right). The bottom figure shows the convergence of the approximation error for $n=64,128$ with respect to the Tucker tensor rank. The line for $n=256$ (not shown) repeats the one for $n=128$. 

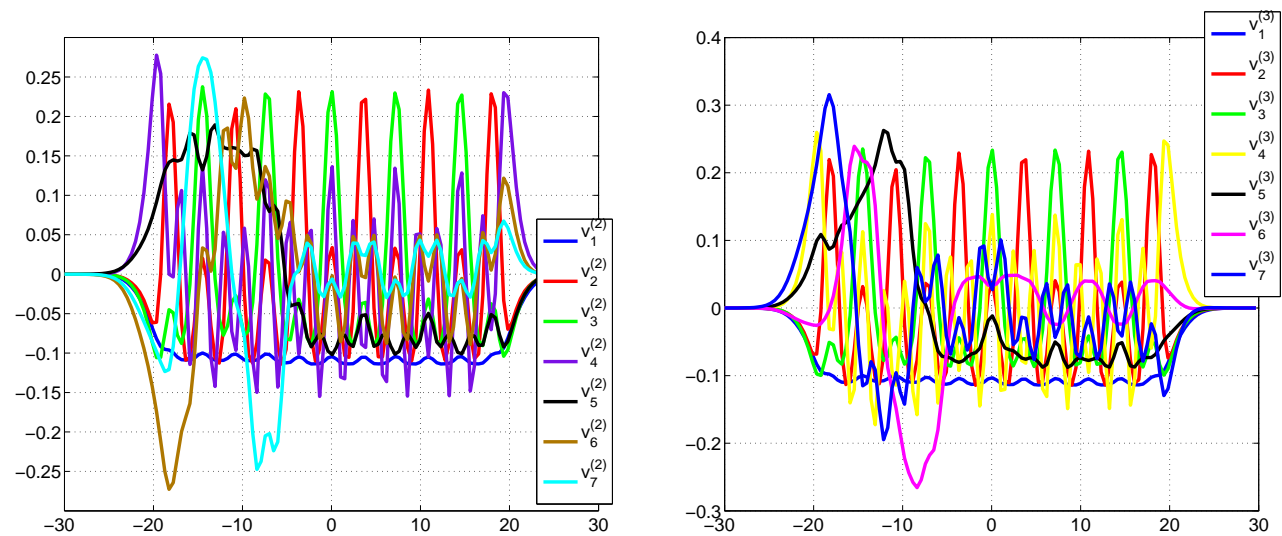

Figure 9: Examples of the first seven Tucker orthogonal vectors in mode 2 (left) and mode 3 (right) for the cluster with 646 Aluminium atoms and randomly located 10 Lithium substitutional atoms and 10 vacancies.

to plane-waves. Moreover, a small Tucker rank is sufficient for accurately representing the electron density obtained by finite element computations. This dramatically reduces the number of degrees of freedom to

$$
O\left(r^{3}+3 r n\right)
$$

which in turn reduces the storage requirements and can reduce the computational complexity of electronic structure calculations. For example, for cluster3 and the Tucker rank $r=10$ and the 3D grid with one-dimension size $n=200$, we need only $N_{\text {storage }}=7000$ entries, in contrast to approximately $20 \mathrm{MB}$ of the original data. This is illustrated in the table 1 below, also for the other clusters. The memory requirements of the Tucker representation reported in table 1 agree with Formula (16) when setting $r=10$ and with the maximal $n$ as stated in Fig. 6. Finally and most importantly, the Tucker rank is almost independent of the system size. A Tucker rank of $r=10$ suffices to approximate the electronic structure of varying system sizes including a single Aluminium atom and clusters containing 14, 172, 365, and 666 atoms as well as a cluster with a vacancy. This suggests that in the Tucker representation, the number of basis functions required to approximate the electronic structure to a desired accuracy only weakly depends on the system size for the aforementioned systems. Further, a Tucker rank of $r=20$ is sufficient 
to represent the electronic structure of a complex system comprising of 646 Aluminium atoms, 10 Lithium substitutional atoms and 10 vacancies.

\begin{tabular}{|c|r|r|c|c|}
\hline Cluster & Atoms & Finite Elements & Memory full tensor & Memory Tucker repr. \\
\hline \hline 1 & 14 & $8,646,656$ & $9438 \mathrm{~KB}$ & $49.3 \mathrm{~KB}$ \\
3 & 172 & $20,643,840$ & $21690 \mathrm{~KB}$ & $50.2 \mathrm{~KB}$ \\
4 & 365 & $39,456,768$ & $39593 \mathrm{~KB}$ & $58.7 \mathrm{~KB}$ \\
5 & 666 & $57,716,736$ & $59685 \mathrm{~KB}$ & $64.3 \mathrm{~KB}$ \\
\hline
\end{tabular}

Table 1: Comparison of memory requirements for storing the full tensor representation and the Tucker approximation with $r=10$ of the electron density and different clusters

\section{Discussion of the results and outlook}

In this article we demonstrated the effectiveness of the Tucker tensordecomposition for the rank-structured representation of the electron density obtained from finite element computations for large Al clusters (also without periodicity). As main result of our numerical investigations, after exploring varying sizes of the Aluminum clusters with repeating FCC unit cells- $1 \times 1 \times 1$ FCC unit cell to $5 \times 5 \times 5$ FCC unit cells-we demonstrated that a small Tucker rank $r$ is sufficient to represent this data, and $r$ is almost independent of the system size. Further, for a complex system where 20 Aluminum atoms in a $5 \times 5 \times 5$ Aluminum cluster are replaced at random with 10 vacancies and 10 Lithium atoms, a Tucker rank $r=20$ is sufficient to approximate the electron density. Hence, the application of the tensor-structured approach with the Tucker representation of the electron density can dramatically reduce the complexity of the problem. This result is very important since it justifies the development of quantum mechanical algorithms (for OFDFT, and possibly KSDFT) based entirely on the Tucker representation and including the computation of all essential parts of the energy functional like nuclear potential, exchange-correlation energy or kinetic energy by the tensor-structured techniques.

Motivated by these results, it seems plausible that the tensor-structured numerical algorithms can be applied further to a large class of applications from physics and chemistry. The pending systematic steps are an error analysis between full data and the reduced representation, and the complete 

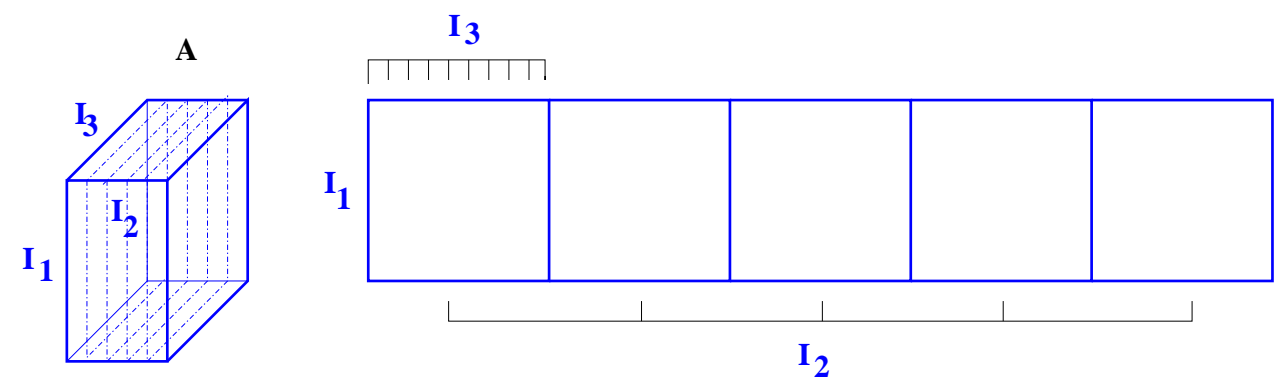

$\mathbf{A}_{(\mathbf{1})}$

Figure 10: Unfolding of the 3 rd order tensor over the mode $\ell=1$ to a matrix $A_{(1)}$ of size $I_{1} \times I_{3} I_{2}$.

implementation of the Kohn-Sham problem in Tucker tensor format. One of the desirable final objectives of the tensor methods based on separable rank-structured representations is the investigation of dislocations and other defects in a non-periodic quantum mechanical setup. Studies of dislocations in a periodic setting can be found in [3], [13]. The far-reaching applications of such a method would include the study of dislocations, vacancies, cracks, doped systems and free surfaces in solids where the core of the defect and the long-ranged elastic effects are studied using quantum mechanics.

\section{Appendix}

Here we describe the unfolding and contraction of the multidimensional tensors [21] for the case $d=3$, though all considerations are valid for $d>3$.

In the Tucker decomposition algorithm we use the unfolding of a tensor into a matrix. The unfolding of a $3 \mathrm{D}$ tensor $A=\left[a_{i_{1} i_{2} i_{3}}\right] \in \mathbb{R}^{I_{1} \times I_{2} \times I_{3}}, I_{\ell}=$ $\left\{1, \ldots, n_{\ell}\right\}$, with respect to the mode $q(\ell, q=1,2,3)$, is denoted by the matrix $A_{(q)}$ of size $n_{q} \times \bar{n}_{q}$, where $\bar{n}_{q}=\prod_{\ell=1, \ell \neq q}^{3} n_{\ell}$. Hence, one of the sizes of the matrix $A_{(q)}$ is the product of all dimension sizes of the tensor $A$ except the $q$-th mode,

$$
A_{(q)}=\left[a_{i_{q} k}\right], \text { with } k=1, \ldots, \bar{n}_{q}, \bar{n}_{q}=\prod_{\ell=1, \ell \neq q}^{3} n_{\ell} .
$$

Figure 10 presents the example of the matrix unfolding over the mode $\ell=1$ for the 3rd order tensor. 
Another important tensor operation is the contracted product of two tensors. In the following we use a tensor-matrix contracted product along mode $\ell$. Given a tensor $A \in \mathbb{R}^{I_{1} \times I_{2} \times I_{3}}$ and a matrix $M \in \mathbb{R}^{J_{\ell} \times I_{\ell}}$, we define the respective mode- $\ell$ tensor-matrix product by ${ }^{1}$

$$
U=A \times_{\ell} M \in \mathbb{R}^{I_{1} \times \ldots \times I_{\ell-1} \times J_{\ell} \times I_{\ell+1} \times \ldots \times I_{d}},
$$

where

$$
u_{i_{1} \ldots i_{\ell-1} j_{\ell} i_{\ell+1} \ldots i_{d}}=\sum_{i_{\ell}=1}^{n_{\ell}} v_{i_{1} \ldots i_{\ell-1} i_{\ell} i_{\ell+1} \ldots i_{d}} m_{j_{\ell} i_{\ell}}, \quad j_{\ell} \in J_{\ell} .
$$

\section{Acknowledgements}

The authors gratefully acknowledge Prof. Dr. M. Ortiz (CalTech, USA), Prof. Dr. W. Hackbusch (MPI MIS, Leipzig), PD Dr.Sci. B. Khoromskij (MPI MIS, Leipzig), and Mr. G. R. Balachandran (Univ. of Michigan, USA) for the valuable comments and useful discussions. V.G. gratefully acknowledges the support of the Air Force Office of Scientific Research under Grant no. FA9550-09-1-0240.

\section{References}

[1] J. Bey, R. Aachen, Simplicial grid refinement: on Freudenthal's algorithm and the optimal number of congruence classes, Numer. Math $\mathbf{8 5}$ (2000), 1-29.

[2] D.M. Ceperly, B.J. Alder, Ground state of the electron gas by a stochastic method, Phys. Rev. 45 (1980), 566-569.

[3] G. Csanyi, S. Ismail-Beigi, T.A. Arias, Paramagnetic structure for the soliton of the $30^{\circ}$ partial dislocation in silicon, Phys. Rev. Letters $\mathbf{8 0}$ (1998), 3984-3988.

[4] L. De Lathauwer, B. De Moor and J. Vandewalle, On the best rank-1 and rank- $\left(R_{1}, \ldots, R_{N}\right)$ approximation of higher-order tensors, SIAM J. Matrix Anal. Appl. 21 (2000), 1324-1342.

\footnotetext{
${ }^{1}$ Note that the order of indices $J_{\ell} \times I_{\ell}$ corresponds to the notation of the Tucker decomposition in (8).
} 
[5] L. De Lathauwer, B. De Moor, J. Vandewalle, A multilinear singular value decomposition, SIAM J. Matrix Anal. Appl. 21 (2000), 1253-1278.

[6] C. Fiolhais, J. P. Perdew, S. Q. Armster, J. M. MacLaren, and M. Brajczewska, Dominant density parameters and local pseudopotentials for simple metals, Phys. Rev. B. 51 (1995) 14001-14011.

[7] M. Finnis, Interatomic Forces in Condensed Matter, Oxford University Press, New York, 2003.

[8] V. Gavini, J. Knap, K. Bhattacharya, and M. Ortiz, Non-periodic Finiteelement formulation of orbital-free density-functional theory, J. Mech. Phys. Solids 55 (2007), 669-696.

[9] V. Gavini, K. Bhattacharya, and M. Ortiz, Quasi-continuum orbital-free density-functional theory: A route to multi-million atom non-periodic DFT calculation, J. Mech. Phys. Solids 55 (2007), 697-718.

[10] W. Hackbusch, B.N. Khoromskij, Tensor-product Approximation to Operators and Functions in High dimension, J. Complexity 23 (2007), 697714.

[11] W.J. Hehre, R.F. Stewart, J.A. Pople, Self-consistent molecular-orbital methods. I. Use of gaussian expansions of slater-type atomic orbitals, J. Chem. Phy. 51 (1969), 2657-2664.

[12] P. Hohenberg, W. Kohn, Inhomogeneous electron gas, Phys. Rev. 136 (1964), B864-B871.

[13] S. Ismail-Beigi, and T.A. Arias, Ab initio study of screw dislocations in Mo and Ta: A new picture of plasticity in bcc transition metals, Phys. Review Letters 84 (2000), 1499-1504.

[14] V. Khoromskaia. Numerical solution of the Hartree-Fock equation by multilevel tensor-structured methods. PhD Dissertation. TU Berlin, 2010. http://opus.kobv.de/tuberlin/volltexte/2011/2948/

[15] B.N. Khoromskij, Fast and Accurate Tensor Approximation of Multivariate Convolution with Linear Scaling in Dimension. J. Comp. Appl. Math., 234 (2010) 3122-3139. 
[16] B.N. Khoromskij, On Tensor Approximation of Green Iterations for Kohn-Sham equations, Computing and Visualisation in Science 11 (2008), 259-271.

[17] B.N. Khoromskij, V. Khoromskaia, Low Rank Tucker Tensor Approximation to the Classical Potentials, Central European J. Math. 5(3) (2007), 1-28.

[18] B.N. Khoromski, V. Khoromskaia, Multigrid Accelerated Tensor Approximation of Function Related Multi-dimensional Arrays. SIAM J. Sci. Comput. 31 (2009), 3002-3026.

[19] B. N. Khoromskij, V. Khoromskaia, H.-J. Flad, Numerical Solution of the Hartree-Fock Equation in the Multilevel Tensor-structured Format, SIAM J. Sci. Comp., v. 33(1), 2011, pp. 45-65.

[20] W. Kohn, L.J. Sham, Self-consistent equations including exchange and correlation effects, Phys. Rev. 140 (1965), A1133-A1138.

[21] T.G. Kolda and B.W. Bader, Tensor Decompositions and Applications, Technical Report Number SAND2007-6702, Sandia National Laboratories, Albuquerque, NM and Livermore, CA, November 2007.

[22] G. Kresse, J. Furthmüller, Efficient iterative schemes for ab initio totalenergy calculations using a plane-wave basis set, Phys. Rev. B 54 (1996), 11169-11186.

[23] R.G. Parr, W. Yang, Density-Functional Theory of Atoms and Molecules, Oxford University Press, New York, 1989.

[24] J.E. Pask, P.A. Sterne, Finite element methods in ab initio electronic structure calculations, Model. Simul. Mater. SC. 13 (2005), R71-R96.

[25] J.P. Perdew, A. Zunger, Self-interaction correction to density-functional approximation for many-electron systems, Phys. Rev. B 23 (1981), 50485079 .

[26] P. Suryanarayana, V. Gavini, T. Blesgen, K. Bhattacharya, and M. Ortiz, Non-periodic finite-element formulation of Kohn-Sham Density Functional Theory, J. Mech. Phys. Solids 58 (2010), 256-280. 
[27] L.R. Tucker, Some mathematical notes on three-mode factor analysis, Psychometrika 31 (1966), 279-311.

[28] Y.A. Wang, N. Govind, E.A. Carter, Orbital-free kinetic-energy density functionals with a density-dependent kernel, Phys. Rev. B. 60 (1999), 16350-16358.

[29] L.W. Wang, M.P. Teter, Kinetic-energy functional of the electrondensity, Phys. Rev. B 45 (1992), 13196-13220. 